\title{
Individual differences in working memory capacity and the regulation of arousal
}

\author{
Matthew K. Robison ${ }^{1}$ (D) . Gene A. Brewer ${ }^{1}$ \\ Published online: 10 July 2020 \\ (C) The Psychonomic Society, Inc. 2020
}

\begin{abstract}
Previously it has been theorized that differential functioning of the locus coeruleus-norepinephrine (LC-NE) system affects people's ability to regulate arousal, which has impacts on cognitive abilities. In the present study, we investigated three potential mechanisms by which the LC-NE system can fail to regulate arousal appropriately: hypoarousal, hyperarousal, and dysregulation of arousal. Each of these three could potentially account for why arousal affects cognition. To test the contributions of these three mechanisms, the present study examined individual differences in working memory capacity (WMC) and the regulation of arousal using pupillometry. Participants completed multiple complex span and visual arrays change-detection measures of WMC. An eye-tracker recorded pupil diameter as participants completed the visual arrays tasks. We found rather mixed evidence for the three mechanisms. Arousal dysregulation correlated with lower visual arrays performance and more self-reported attentional lapses. However, arousal regulation did not correlate with complex span performance. There was also some evidence for hypoarousal as an explanatory mechanism, as arousal correlated with attentional lapses. We discuss the implications of the results for theories regarding the role of arousal regulation in cognitive performance and individual differences in cognitive abilities.
\end{abstract}

Keywords Working memory capacity · Attention control · Arousal · Locus coeruleus · Norepinephrine

Working memory capacity (WMC), the ability to maintain, manipulate, and retrieve goal-relevant information, is a core cognitive construct. Decades of research have reinforced this idea, demonstrating that individual differences in WMC correlate with important outcomes like fluid intelligence (Conway, Cowan, Bunting, Therriault, \& Minkoff, 2002; Engle, Tuholski, Laughlin, \& Conway, 1999; Kane et al., 2004; Kyllonen \& Christal, 1990), reading comprehension (Daneman \& Carpenter, 1980; McVay \& Kane, 2012b; Unsworth \& McMillan, 2013), learning (Kyllonen \&

We would like to thank Alaina Dettmer, Haitham Shakir, Cyril Abadir, Kameron Moore, Taylor Rhea, and Fransisco Herold for their assistance with data collection. We would also like to thank Nash Unsworth and Jason Tsukahara for their helpful commentary on earlier versions of this manuscript. Finally, we would like to thank Stephen Goldinger for providing the equipment necessary to complete this project. The authors were funded by National Science Foundation grant 1632327.

Matthew K. Robison

matthewkrobison@asu.edu

1 Department of Psychology, Arizona State University, 950 S. McAllister Ave., Tempe, AZ 85287, USA
Stephens, 1990; Unsworth \& Engle, 2005), and academic aptitude tests (Engle et al., 1999; Unsworth et al., 2012a, b). A great deal of research has investigated precisely why WMC predicts such a wide variety of outcomes. According to the executive-attention account of WMC, the predictive power of WMC derives from its substantial overlap with attention control (Engle \& Kane 2004; Kane, Bleckley, Conway, \& Engle, 2001; Kane \& Engle, 2002). Here, attention control is conceptualized as the set of processes that allow for the selection and activation of task goals and selection of task-relevant information in the presence of internally and externally distracting sources of information. Thus, WMC predicts outcomes (e.g., reading comprehension) because both WMC and the respective outcomes require a certain degree of attention control in order to perform such tasks well.

Another conceptualization of WMC is the sheer amount of information that individuals can maintain in a prioritized state (Cowan, 2001; Luck \& Vogel, 1997). This account holds that people with high WMC tend to demonstrate greater cognitive performance because they can manage more information at one time (e.g., Fukuda, Vogel, Mayr, \& Awh, 2010; Cowan et al., 2005). Subsequent theories have 
argued that WMC is a constellation of attention control, short-term memory capacity, and controlled retrieval from long-term memory (Shipstead, Harrison, \& Engle, 2015; Shipstead, Lindsey, Marshall, \& Engle, 2014; Shipstead, Redick, Hicks, and Engle (Shipstead et al., 2012); Unsworth 2016, Unsworth \& Engle, 2007a, b; Unsworth et al., 2014), and each of these abilities separately account for the shared variance between WMC and higher-order cognition (e.g., fluid intelligence). These lines of research have focused heavily on cognitive mechanisms which account for WMCrelated differences in behavior. But aside from cognitive mechanisms, another set of important and unanswered question remains. Given there is such substantial variation in WMC even among relatively healthy adults, where does this variation come from? At the level of neural mechanisms, what causes some people to have higher WMC than others?

One prominent theory is that variation in WMC derives from differences in the functioning of the prefrontal cortex, specifically the dorsolateral prefrontal cortex (DLPFC). Drawing upon an impressive array of research involving human and animal lesion studies, individual differences investigations, and human neuroimaging work, Kane and Engle (2002) showed that the DLPFC is especially active during tasks that require the putative cognitive mechanisms required by the working memory system. Further, damage to this area or temporary inactivation by external agents inhibits an organism's ability to perform such operations. Thus, Kane and Engle (2002) theorize that the relative functioning of the DLPFC underlies individual differences in $\mathrm{WMC}$ and attention control.

Recently, Unsworth and Robison (2017a) proposed a different neural mechanism: dysregulation of the locus coeruleus-norepinephrine (LC-NE) system. The LC is a brainstem nucleus whose neurons release the majority of cortical NE (Berridge \& Waterhouse, 2003). It has dense projections into most of cortex, and it has both afferent and efferent connections with areas that have theoretical roles for attention and working memory (e.g., PFC). Historically, it has been argued that the LC has a general role in promoting wakefulness, alertness, and arousal, but more recently is has been shown that the LC has more specific functional connections (see Berridge and Waterhouse (2003), Samuels and Szabadi (2008), and Szabadi (2013) for reviews).

Aston-Jones and Cohen (2005)'s influential Adaptive Gain Theory of the LC-NE system argues for a rather specific role of the LC in regulating attention. Aston-Jones and Cohen note that the LC exhibits two modes of firing. The first is a slow, rhythmic firing $(1-3 \mathrm{~Hz})$ that presumably regulates a level of tonic arousal in the organism. The second is a rapid, phasic burst of firing in response to the perception of a goal-relevant stimulus in the environment.
Importantly, there is an interdependence between these two aspects of activity. When tonic firing is low, the organism is in a hypoaroused, drowsy state, and the system does not demonstrate marked phasic firing to nearly any stimulus. At an intermediate level of tonic firing, phasic firing is highest and most selective. Thus, during goal-directed behavior, it is often optimal for the organism to be in this mode, which Aston-Jones and Cohen call the "exploitative" mode. The organism selectively processes relevant stimuli in order to achieve some goal (e.g., detect prey, avoid predators, earn rewards). At even higher levels of tonic activity, the organism is in a hyperaroused, overactive state. The system responds to stimuli rather indiscriminately. In some cases, this can be advantageous. Aston-Jones and Cohen refer to this mode as "explorative". It can allow the organism to seek new environments and possibilities for rewards. However, when there is a specific goal, hyperarousal can be harmful. Thus, there is a trade-off between exploration and exploitation, and the system must adapt to the situation in order to match tonic activity with the environmental and situational demands.

Aston-Jones and Cohen (2005) argue that the functional consequence of phasic firing of the $\mathrm{LC}$ is to release $\mathrm{NE}$ into the cortical regions responsible for the goal-relevant computation. NE has a gain-modulating role on target cortical neurons. It enhances firing among neurons that code for goal-relevant stimuli and inhibits firing among neurons that code for irrelevant stimuli, thus increasing the signal-tonoise ratio. So in a way, the LC-NE system directs attention and target cortical regions implement it.

The LC-NE account of individual differences in WMC draws upon many of the assumptions and theoretical mechanisms proposed by Aston-Jones and Cohen. Additionally, it draws upon work on large-scale brain networks, specifically the fronto-parietal control network (FPN), the salience network (SN), and the default-mode network (DMN). As the name suggests, the FPN is a collection of cortical areas that are particularly active during a wide variety of cognitively demanding tasks that require maintenance of task goals in the presence of interference and distraction (Cohen, Aston-Jones, \& Gilzenrat, 2004; Corbetta \& Shulman, 2002; Dosenbach, Fair, Cohen, Schlaggar, \& Petersen, 2008; Fox et al., 2005; Miller \& Cohen, 2001; Petersen \& Posner, 2012; Vincent, Kahn, Snyder, Raichle, \& Buckner, 2008). The default-mode network (DMN) consists of a broad collection of brain regions, including the medial prefrontal cortex, lateral frontal cortex, medial parietal cortex, medial temporal lobe, and lateral temporal cortex. Of note is the fact that the DMN becomes particularly active during periods of internally directed cognition, such as mind-wandering and autobiographical retrieval (AndrewsHanna, Smallwood, \& Spreng, 2014; Raichle et al., 2001). Thus when the task calls for internally directed cognition, 
the DMN and FPN work in concert. However, during effective execution of externally directed tasks requiring attention control, the FPN and DMN are anticorrelated (Konishi, McLaren, Engen, \& Smallwood, 2015; Smallwood et al., 2013; Spreng et al., 2014; Vatansever, Menon, Manktelow, Sahakian, \& Stamatakis, 2015). The salience network (SN) comprises the dorsal anterior cingulate cortex and the frontal operculum/anterior insula cortex. Theoretically, the $\mathrm{SN}$ is responsible for identifying motivationally salient and goal-relevant stimuli (Menon \& Uddin, 2010; Seeley et al., 2007). Importantly, one if its functions appears to be the marking of stimuli for further processing by other cortical regions (e.g., the FPN) while disengaging cortical regions that process goal-irrelevant information (e.g., the DMN).

The theory posits a role of the LC in the functional relationship of these large-scale networks. Specifically, it argues that consistent and appropriate release of $\mathrm{NE}$ from the LC activates the FPN and deactivates the DMN so task-relevant computations can be performed without interference. When the $\mathrm{SN}$ detects an error or a particularly slow response, it sends a signal to the LC to adjust tonic activity accordingly. Unsworth and Robison propose that one source of variation in WMC is thus a breakdown of this system. When the system does not function optimally, the LC does not regulate arousal levels properly. States of hypoor hyperarousal allow the DMN to become active, resulting in attentional lapses. Evidence for the theory comes from both behavioral and physiological measures.

Behaviorally, people with low WMC tend to experience more attentional lapses during tasks that require a consistent allocation of attention. Indeed, this is one of the central aspects of the executive-attention account of WMC. For example, during simple reaction time tasks, high- and lowWMC participants perform equally on the majority of trials. However, people with low-WMC tend to experience occasional trials where they respond particularly slowly (Unsworth, Redick, Lakey, \& Young, 2010). Similar results are found with choice reaction time tasks, as the relationships between WMC and speed are largest for the slowest trials (Schmiedek, Oberauer, Wilhelm, Süß, \& Wittmann, 2007). Theoretically, these instances reflect lapses of attention. Furthermore, a growing literature has demonstrated that people with relatively low WMC tend to report more attentional lapses in the form of mindwandering and distraction, especially during cognitively demanding tasks (Kane et al., 2016, 2007; McVay \& Kane, 2012a, b; Robison et al. ,2017; Robison \& Unsworth, 2015, 2018; Unsworth \& McMillan, 2013, 2014). Thus, there is converging evidence that one central aspect of WMCrelated variation is the ability to consistently apply attention toward externally directed tasks. Unsworth and Robison argue that this variation arises because of a breakdown in the
LC-NE system's ability to keep a person in an intermediate state of arousal. As described earlier, Aston-Jones and Cohen (2005) argue that both hyper- and hypoarousal can cause indiscriminate or very little overall responding to stimuli, respectively. Thus, when people cannot maintain a consistent level of tonic arousal, they will experience attentional lapses (e.g., mind-wandering and distraction), and thus experience poor cognitive performance.

For reasons that are still not entirely mapped out, pupil diameter can be used as a close temporal correlate of the firing of LC neurons. Decades of research using direct recordings from animals, including non-human primates, has demonstrated this link (Aston-Jones \& Cohen, 2005; Eldar, Cohen, \& Niv, 2013; Gilzenrat, Nieuwenhuis, Jepma, \& Cohen, 2010; Joshi, Li, Kalwani, \& Gold, 2016; Samuels \& Szabadi, 2008; Varazzani, San-Galli, Gilardeau, \& Bouret, 2015). Importantly, pupil diameter can be used to measure both tonic and phasic activity of the LC. In the present study, we leverage this connection by using pupil diameter as an indirect measure of tonic activity in the LC (i.e., arousal). Our primary dependent measure was pretrial pupil diameter, which presumably reflects a momentary state of arousal. From this trial-by-trial measure, we examined mean levels of arousal and intraindividual variability in arousal.

The LC-NE account makes several predictions regarding individual differences in task performance, self-reporting of attentional lapses, and pupillary measures. First, it makes the prediction that individuals with low WMC will show worse performance on tasks that require a consistent allocation of externally directed attention toward a goal. Second, it makes the prediction that participants with low WMC will report more attentional lapses (e.g., mind-wandering, external distraction) during such tasks. Third, it makes the prediction that people with low WMC will experience more fluctuations in arousal (i.e., higher intraindividual variability) during such tasks. Fourth, it makes the prediction that low WMC people will exhibit lower task-evoked (i.e., phasic) pupillary responses, on average.

In prior work, Unsworth and Robison have found evidence for these relationships. In one study, Unsworth and Robison (2015) examined pretrial and phasic pupillary measures while participants completed a visual arrays task. Participants who performed well on the visual arrays task exhibited less variability in pretrial pupil diameter and greater phasic responses. Mean pretrial pupil diameter did not correlate with WMC ( $k$ estimate from the visual arrays task). Variability in arousal and average phasic responding both accounted for significant portions of variance in $k$, suggesting these are dissociable individual differences that both have implications for WMC. In a second study, participants in several different experiments 
completed various versions of visual arrays tasks (Unsworth \& Robison, 2018). Although the individual experiments did not have enough participants for an adequate analysis of individual differences, Unsworth and Robison combined data across experiments to do so. They estimated $k$ for each participant based on their performance on the tasks. Then, they examined correlations between $k$ and changes in phasic pupillary responses as a function of set size. High- $k$ individuals showed larger changes in phasic pupil responses as set size increased. In a third study, Robison and Unsworth examined pupillary measures during the course of a visual arrays task in three experiments (Robison \& Unsworth, 2019). Again, they did not have sufficient power to examine individual differences in each individual experiment. But combining data across experiments, they examined correlations among $k$, pretrial pupil diameter, and phasic pupillary responses. High$k$ participants exhibited less variability in pretrial pupil diameter, greater overall phasic pupillary responses, and less variability in phasic pupillary responses. Mean pretrial pupil diameter did not correlate with $k$. In a fourth study, participants completed a battery of complex span working memory and attention control tasks during a single laboratory session (Unsworth \& Robison, 2017b). They measured pupil diameter while participants completed the attention control tasks. Using confirmatory factor analysis, they derived latent variables for WMC, attention control, average tonic arousal, variability in tonic arousal, average phasic responses, and variability in phasic responses. WMC positively correlated with attention control and negatively correlated with attentional lapses, average pretrial pupil diameter, and variability in pretrial pupil diameter. Further, WMC negatively correlated with variability in phasic pupillary responses (but not average phasic responses).

Other researchers have also investigated the link between pupil measures and WMC. For example, Heitz, Schrock, Payne, and Engle (2008) measured pupil diameter for highand low-WMC participants. The high-WMC participants (upper quartile of distribution) had larger pre-experimental pupil diameters and higher baseline pupil diameter across trials than low-WMC participants (lower quartile of distribution). Even after an incentivization manipulation, lowWMC participants showed smaller pupil diameters than their high-WMC counterparts. In a recent study, Tsukahara, Harrison, and Engle (2016) examined relationships between baseline (pre-experimental and pretrial) pupil diameter and WMC. Across several experiments, Tsukahara et al. observed significant differences between highand low-WMC individuals, as well as people who differed in fluid intelligence, in pupil diameter. They interpreted their findings to indicate that people vary in cognitive ability because of differences in the functional organization of the resting-state brain arising from the neuromodulatory role of the LC-NE system. Specifically, they argue that a disruption of the functional connectivity between the LC, the DMN, and FPN is one cause of poor cognitive performance during situations that require an external allocation of attention (e.g., WMC and fluid intelligence tasks). Although Tsukahara et al. (2016) do not make this specific claim, this finding would be consistent with people low in cognitive ability being relatively hypoaroused (see also Heitz et al., 2008).

Another possibility is that low-WMC individuals tend to be hyperaroused. Via this explanation, low-WMC individuals' higher arousal leads to lapses of attention, and their cognitive performance suffers as a consequence. If this is the case, we should observe a negative correlation between WMC and pretrial pupil diameter. In their latent variable analysis, Unsworth and Robison (2017b) observed a negative correlation between mean baseline pupil diameter and WMC. So there is some evidence for this account. Finally, the LC-NE account of WMC discussed above (Unsworth \& Robison, 2017a) argues that dysregulation of arousal is the major factor linking LCNE system functioning to cognitive ability. The hypoarousal and hyperarousal accounts are not necessarily mutually exclusive with the dysregulation account. That is, it is possible that low-WMC individuals tend to be hypoaroused (or hyperaroused) and experience more volatility in arousal. Using structural equation modeling, we will be able to test for these possibilities. Table 1 summarizes the predictions that each plausible mechanism makes for the present study, and Fig. 1 shows a graphical depiction of the hypotheses.

\section{The present study}

In many ways, the present study was designed as a followup and extension to Unsworth and Robison (2017b). In that study, Unsworth and Robison (2017b) measured WMC and attention control with a battery of tasks. During the attention

Table 1 Theoretical mechanisms and their empirical predictions

\begin{tabular}{ll}
\hline Mechanism & Prediction \\
\hline Hypoarousal & $\begin{array}{l}\text { Average pretrial pupil diameter will positively } \\
\text { WMC correlate with and negatively correlate } \\
\text { with attentional lapses. }\end{array}$ \\
Hyperarousal & $\begin{array}{l}\text { Average pretrial pupil diameter will negatively } \\
\text { correlate with WMC and positively correlate } \\
\text { with attentional lapses. } \\
\text { Dysregulation }\end{array}$ \\
& $\begin{array}{l}\text { Variability (CoV) in pretrial pupil diameter } \\
\text { will negatively correlate with WMC and positively } \\
\end{array}$ \\
& correlate with attentional lapses.
\end{tabular}




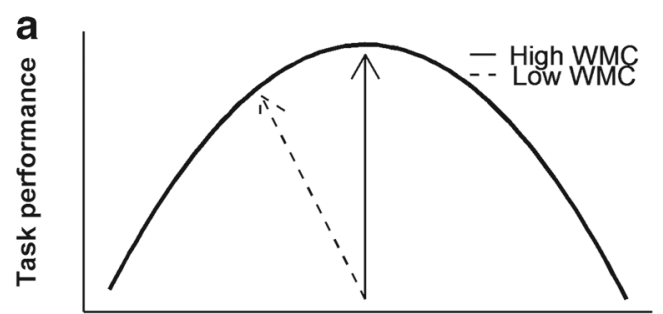

Arousal

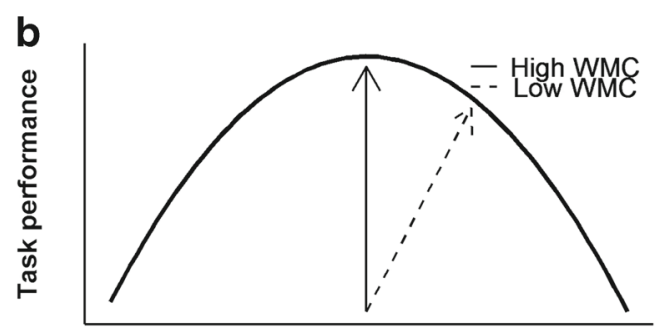

Arousal

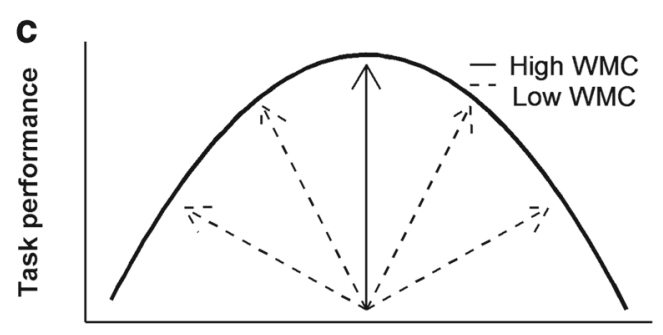

Arousal

Fig. 1 Three potential mechanisms by which individual differences in working memory capacity (WMC) could be associated with arousal. Low-WMC participants could a be chronically hypoaroused, b be chronically hyperaroused, or c experience more volatility in arousal

control tasks, they recorded pupil diameter to measure both tonic (pretrial) and phasic (task-evoked) arousal. They also added thought probes to the attention control tasks to measure subjective states of inattention (e.g., mindwandering and external distraction). Importantly, the pupil and thought probe measures were all collected during the attention control tasks. Here, we wanted to assess whether the pattern of findings would hold if the same measures were included during tasks that are designed to measure WMC. Unsworth and Robison (2017b) used three complex span tasks to measure WMC. There are several elements of complex span tasks that make it difficult to measure and interpret pupil and thought probe data during them. First, "trials" consist of lists that alternate between encoding of memoranda and a distractor task. Thus, any task-evoked pupillary responses will be heavily influenced by changes in luminance across screens and list items. Second, there are relatively fewer trials in which something like pretrial pupil diameter can be measured. Third, inserting thought probes is not as straightforward as it is in attention control tasks because it is unclear exactly where these probes should be included to catch people in off-task attentional states. This would allow us to extend the methodology of measuring attentional lapses and arousal regulation during situations that primarily engage attention control (Unsworth \& Robison, 2017b) to situations that engage an individual's working memory system, as well. Thus, the present study was intended to replicate the pattern of relations observed in Unsworth and Robison (2017b) and extend the methodology to measuring arousal during working memory tasks.

For all these reasons, we decided to include thought probes and pupil measurement during visual arrays (changedetection) working memory tasks, while also measuring WMC with complex span tasks. Prior work has used these visual arrays tasks to examine the pupillary correlates of WMC, but these studies (Robison \& Unsworth, 2019; Unsworth \& Robison, 2015, 2018) had several weaknesses that we aimed to address in the present study. First, the prior studies used single tasks to measure WMC and they did not have externally validating measures. Second, because we used single tasks, we could not assess whether the behavioral and pupillary measures were task-general or task-specific. Third, in several studies, we make the claim that variability in pupil diameter (i.e., arousal) is indicative of attentional lapses. However, in most of those studies we did not measure such lapses. Thus, the current study is a combination of the latent-variable approach taken by Unsworth and Robison (2017b) with the pupillary approaches taken to studying working memory with visual arrays tasks (Robison \& Unsworth, 2019; Unsworth \& Robison, 2015, 2018).

We continuously recorded pupil diameter during the visual arrays tasks. Each of the visual arrays tasks took about $25 \mathrm{~min}$ to complete, allowing us to examine variability in arousal across time. Additionally, we included thought probes to measure attentional lapses throughout the visual arrays tasks. For the reasons described above, we only included thought probes and measured pupil diameter during the visual arrays tasks. For each participant and for each task, we computed mean pretrial pupil diameter (in millimeters) and the coefficient of variation $(\mathrm{CoV})$ in pretrial pupil diameter. Then, we used confirmatory factor analysis and structural equation modeling to estimate relationships among latent factors representing complex span WM, visual arrays WM, average arousal, variability in arousal, and attentional lapses. This allowed us to accomplish several goals: 1) examine the relationships among WMC and pupillary indices of arousal at the latent level, 2) examine relationship between WMC and the tendency to experience attentional lapses, and 3) test predictions made by the LC-NE account of individual differences in WMC (Unsworth \& Robison, 2017a). 


\section{Method}

\section{Participants and procedure}

A sample of 213 participants (109 women, 104 men, $\left.M_{\mathrm{age}}=18.71, S D_{\mathrm{age}}=1.06\right)$ from the human subject pool at Arizona State University completed the study in exchange for partial course credit. Our goal was to achieve a minimum sample size of 200 participants, and we used the end of the academic term as our stopping rule for data collection. Participants completed all the tasks within a single 2-h session. At the beginning of the session, participants completed informed consent and demographics forms. Participants completed the tasks in the following order: color change-detection, operation span, orientation change-detection, symmetry span, letter change-detection, and reading span. At the end of the session, participants completed a computerized self-report measure of attentiondeficit/hyperactivity disorder symptoms (Kessler et al., 2005). However, these data were collected as pilot data for a separate project, and they are not analyzed in the present study. All participants were treated according to the ethical principles of the American Psychological Association.

\section{Tasks}

\section{Visual arrays}

Color change-detection In this task, participants were required to remember the colors of squares in various spatial locations (Luck \& Vogel, 1997). Each trial began with a 2000-ms fixation screen upon which a black fixation cross was centered on a light grey background. There were three trial types: precue, retrocue, and neutral. On precue trials, the fixation screen was followed by a 250 -ms blank screen, then a 250 -ms directional cue $(<$ or $>$ ), then another 250ms blank screen. Then, an array of six colored squares (each $20 \times 20$ pixels; $1^{\circ}$ visual angle) appeared within a $540 \mathrm{x}$ 402 pixel region centered on the screen for $250 \mathrm{~ms}$. The spatial location of items was random, with the requirement that no item appear within a 100-pixel distance of any other item (measured from each item's upper-leftmost point). The colors of the items were sampled from a list of seven possible colors (red, blue, violet, green, yellow, black, or white). Colors did not repeat within a trial. On retrocue trials, the fixation screen was followed by a 750-ms blank screen, then the stimulus array for $250 \mathrm{~ms}$. After $1750 \mathrm{~ms}$, a directional cue $(<$ or $>)$ appeared for $250 \mathrm{~ms}$. The test array then appeared after another 2000-ms delay. On precue and retrocue trials, three items always appeared on each hemifield of the screen. Cues were $100 \%$ valid. On neutral trials, the fixation screen was followed by a 750-ms blank delay, then the stimuli appeared for $250 \mathrm{~ms}$, then there was a 4000-ms delay before the test. On the test screens for all trial types, the stimuli reappeared with one of the squares surrounded by a black circle. The participant's task was to decide whether that square was the same color or a different color as it had been during the first presentation of the array. Participants pressed a key marked "S" to indicate same or a key marked " $D$ " to indicate different (the F and J keys on the keyboard, respectively). For this task and the other changedetection tasks, we collapsed across trial types to obtain a single accuracy estimate for the task which was then used as the dependent variable in all analyses. Participants first completed five practice trials of each trial type. They then completed 126 experimental trials (42 of each trial type). Thought probes followed 16 randomly sampled trials. The pre- and retrocueing manipulations were included as part of a larger individual differences investigation (Robison, Brewer, \& Unsworth, 2020). This task took about $25 \mathrm{~min}$ to complete. See Fig. 2 for a visualization of the trial sequences. Accuracy across the three trial types showed a high level of internal consistency $(\alpha=0.86)$, so we felt comfortable collapsing across trial types to obtain a single accuracy measure for the task. Also, $k$ scores (set size $\mathrm{x}$ [hit rate - false alarm rate]) highly correlated with the accuracy measure $(r=0.85)$. So we believe these two ways of scoring the data indicated largely the same individual difference.

Orientation change-detection In this task, participants were required to remember the orientations of colored bars in various spatial locations (Vogel, McCollough, \& Machizawa, 2005). The task included three randomly interleaved trial types: neutral trials, precue trials, and retrocue trials. Each trial began with a 2000-ms fixation screen upon which a black fixation cross was centered on a light grey background. On precue trials, the fixation screen was followed by a 250 -ms blank screen, then a $250-\mathrm{ms}$ cue ("blue" in blue font or "red" in red font) centered on the screen. After another 250-ms blank screen, the stimuli appeared. In the neutral and retrocue trials, the prestimulus period was a 750-ms blank delay screen. The stimuli were an array of six oriented rectangles (each $20 \times 60$ pixels; $3^{\circ}$ visual angle) that appeared within a $540 \times 402$ pixel region centered on the screen. The spatial location of items was random, with the requirement that no item appear within a 100-pixel distance of any other item (measured from each item's upper-leftmost point). On every trial, there were three red bars and three blue bars. Each bar could be one of four possible orientations: vertical, horizontal, tilted $45^{\circ}$ right, or tilted $45^{\circ}$ left. Orientations could repeat within a trial. In the neutral and precue trials, the stimuli were followed by a 4000-ms blank delay screen. In the retrocue trials, the cue (red or blue) appeared after $1750 \mathrm{~ms}$ and stayed on-screen for $250 \mathrm{~ms}$. There was then another 2000-ms blank delay screen. See Fig. 3 for a visualization of the 


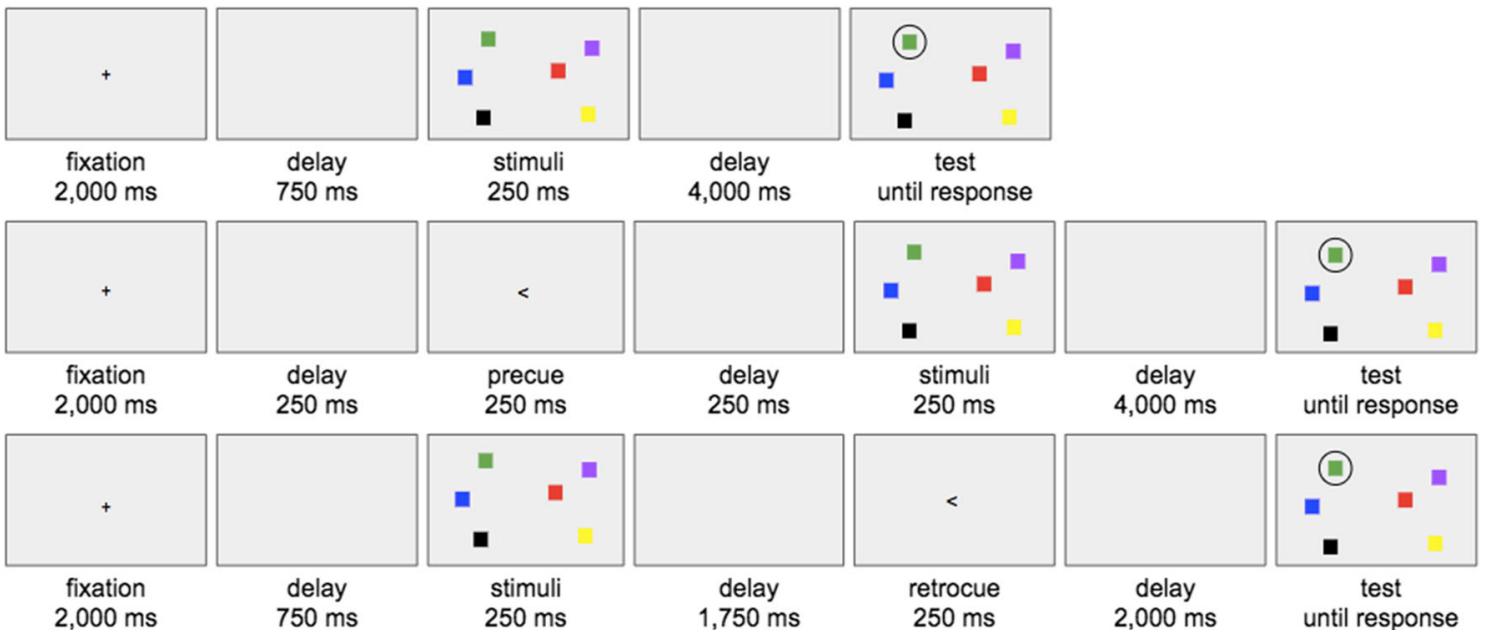

Fig. 2 Trial sequences for three trial types of color change-detection task. The top row shows neutral trials, the middle row shows precue trials, and the bottom row shows retrocue trials. Note: figure is drawn for illustrative purposes and not precisely to scale

trial sequences. At test, the items reappeared. One bar had a white dot on it. The participant's task was to decide whether that bar was the same orientation or a different orientation as it had been during the first presentation of the array. Participants pressed a key marked " $\mathrm{S}$ " to indicate same or a key marked "D" to indicate different (the F and J keys on the keyboard, respectively). The next trial immediately began after participants made their response. Participants completed five practice trials of each trial type. They then completed 126 experimental trials (42 of each trial type). Thought probes followed 16 randomly sampled trials. This task took about 25 min to complete. Accuracy across the three trial types showed a high level of internal consistency $(\alpha=0.87)$, so we felt comfortable collapsing across trial types to obtain a single accuracy measure for the task. $K$ scores also highly correlated with the raw accuracy measure $(r=0.79)$.
Letter change-detection In this task, participants were required to remember the identities of letters that appeared in six fixed spatial locations. Each trial began with a 2$\mathrm{s}$ fixation screen upon which a black fixation cross was centered on a light grey background. The stimuli were six randomly sampled consonants. Letters did not repeat within a trial. The stimuli always appeared in the same six spatial locations, spaced equally around an invisible circle. In this task the pre- and retrocues highlighted three locations by flashing a black box around them. At test, the items reappeared. One letter was outlined by a black box. The participant's task was to decide whether that letter was the same letter or a different letter as it had been during the first presentation of the array. Participants pressed a key marked "S" to indicate same or key marked " $D$ " to indicate different (the F and J keys on the keyboard, respectively). The next trial immediately began after participants made

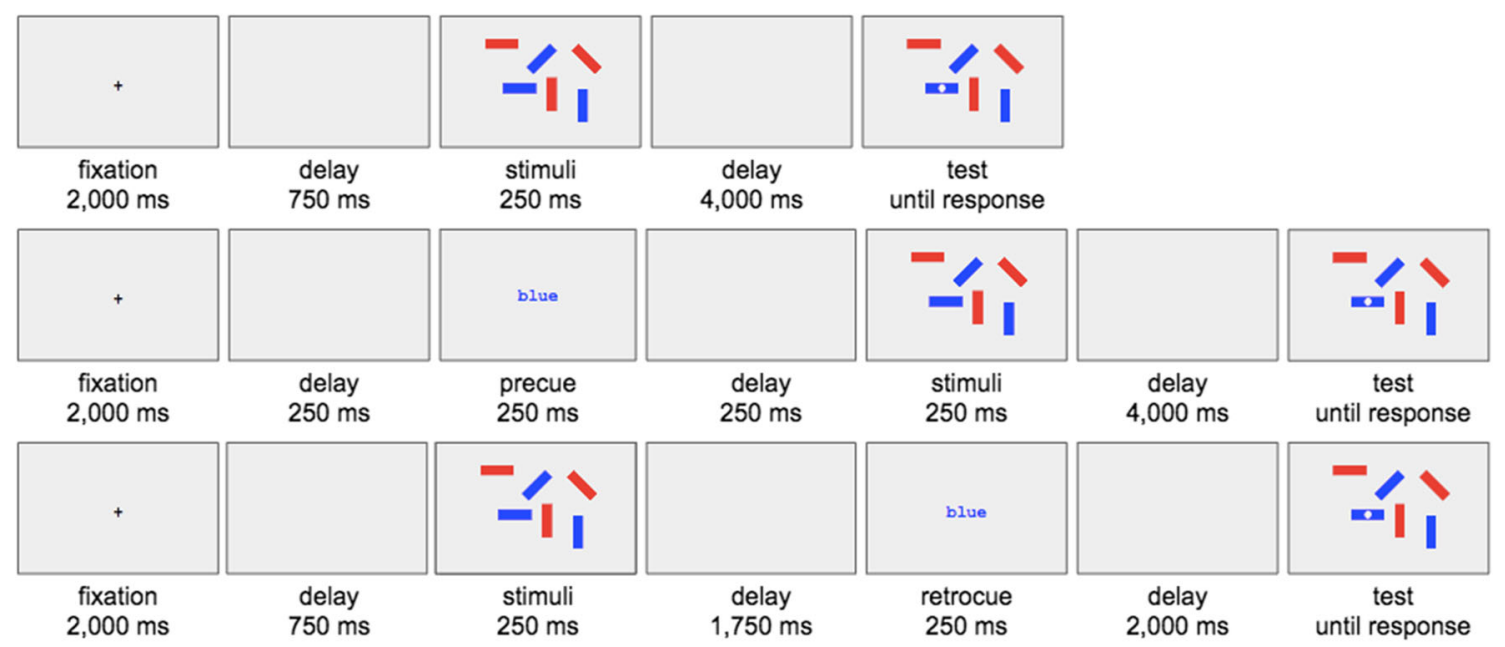

Fig. 3 Trial sequences for orientation change-detection task. The top row shows neutral trials, the middle row shows precue trials, and the bottom row shows retrocue trials. Note: figure is drawn for illustrative purposes and not precisely to scale 
their response. Participants completed five practice trials of each trial type first (neutral, precue, then retrocue). They then completed 126 experimental trials (42 of each trial type). Thought probes followed 16 randomly sampled trials. This task took about 25 min to complete. See Fig. 4 for a visualization of the task sequences. Performance across the three trial-types showed a high-level of internal consistency ( $\alpha=0.86$ ), so we were comfortable collapsing across trial types for a single accuracy measure. $K$ scores for the task also showed a high correlation with raw accuracy $(r=0.82)$.

\section{Thought probes}

In order to measure tendencies to experience attentional lapses, we included thought probes in the color, orientation, and letter change-detection tasks. The thought probes asked participants, "What were you thinking about just prior to when this screen appeared?" The screen had five response options: 1) I was totally focused on the current task, 2) I was thinking about my performance on the task or how long it is taking, 3) I was distracted by sights/sounds around me or by physical sensations (e.g., hungry/thirsty), 4) I was thinking about things unrelated to the task (i.e., mind-wandering), 5) I wasn't thinking about anything/my mind was blank. Participants were instructed to press the key that best described their preceding thoughts. During the instructions to the tasks, participants were told that it is perfectly normal to mind-wander, zone out, or get distracted from time to time on tasks like these, and that they should answer the questions honestly and accurately. For the correlational analyses, we summed reports of mindwandering, external distraction, and mind-blanking and divided by 16 (the total number of probes) into a lapse rate variable.

\section{Complex span}

Operation span In this task, participants solved a series of math operations while trying to remember a set of unrelated letters (Unsworth et al., 2005). Participants were required to solve a math operation, and after solving the operation, they were presented with a letter for $1 \mathrm{~s}$. Immediately after the letter was presented the next operation was presented. At recall participants were asked to recall letters from the current set in the correct order by clicking on the appropriate letters. For all of the span measures, items were scored correct if the item was recalled correctly from the current list in the correct serial position. Participants were given practice on the operations and letter recall tasks only, as well as two practice lists of the complex, combined task. List length varied randomly from three to seven items, and there were two lists of each length for a total possible score of 50. The score was total number of correctly recalled items in the correct serial position. The task took about $12 \mathrm{~min}$ to complete.

Symmetry span Participants recalled sequences of red squares within a matrix while performing a symmetryjudgment task (Unsworth, Redick, Heitz, Broadway, \& Engle, 2009). In the symmetry-judgment task, participants were shown an $8 \times 8$ matrix with some squares filled in black. Participants decided whether the design was symmetrical about its vertical axis. The pattern was symmetrical half of the time. Immediately after determining whether the pattern was symmetrical, participants were presented with a 4 X 4 matrix with one of the cells filled in red for $650 \mathrm{~ms}$. At recall, participants recalled the sequence of red-square locations by clicking on the cells of an empty matrix. Participants were given practice on the symmetry-judgment

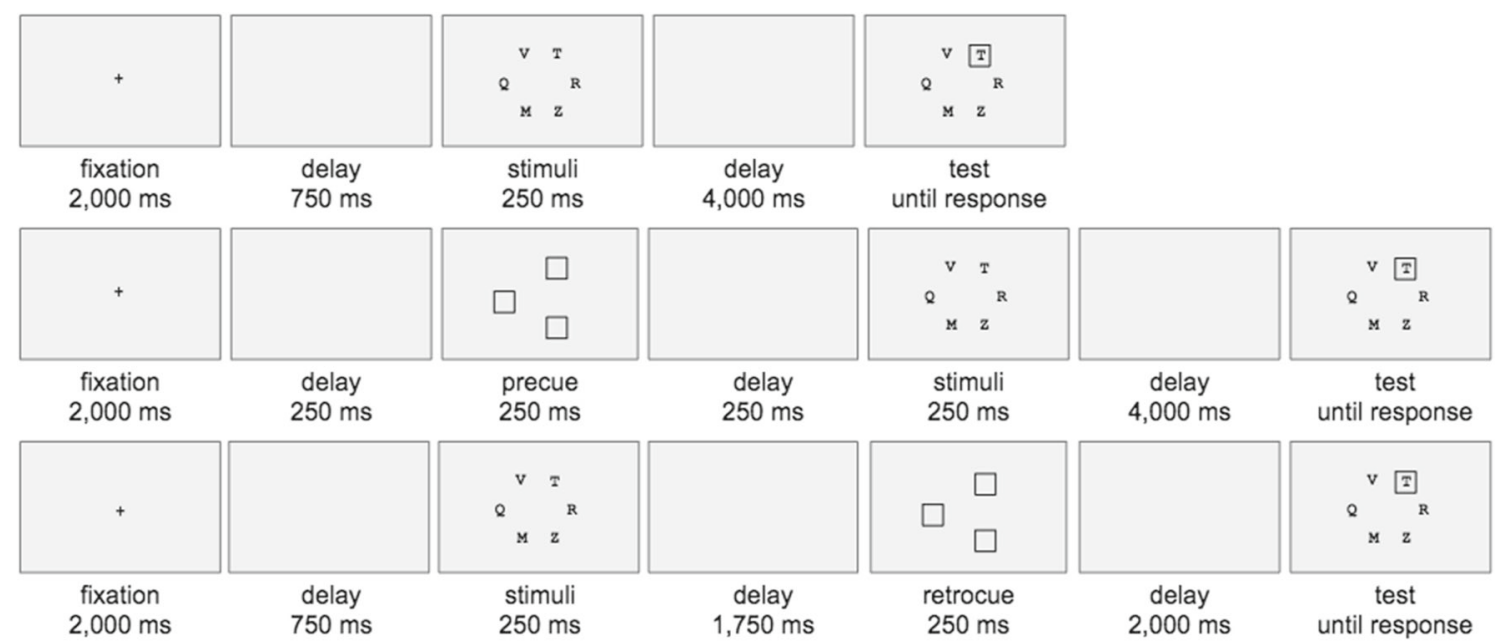

Fig. 4 Trial sequences for letter change-detection task. The top row shows neutral trials, the middle row shows precue trials, and the bottom row shows retrocue trials. Note: figure is drawn for illustrative purposes and not precisely to scale 
and square recall task as well as two practice lists of the combined task. List length varied randomly from two to five items, and there were two lists of each length for a total possible score of 28 . The task took about $10 \mathrm{~min}$ to complete.

Reading span While trying to remember an unrelated set of letters, participants were required to read a sentence and indicated whether or not it made sense (Unsworth et al., 2009). Half of the sentences made sense, while the other half did not. Nonsense sentences were created by changing one word in an otherwise normal sentence. After participants gave their response, they were presented with a letter for 1 s. At recall, participants were asked to recall letters from the current set in the correct order by clicking on the appropriate letters. Participants were given practice on the sentence judgment task and the letter recall task, as well as two practice lists of the combined task. List length varied randomly from three to seven items, and there were two lists of each length for a total possible score of 50 . We used the same scoring procedure as we used in the operation span and symmetry span tasks. The task took about $12 \mathrm{~min}$ to complete.

\section{Pupillometry}

A Tobii T-1750 eye-tracker continuously recorded pupil diameter and gaze position from both eyes at $60 \mathrm{~Hz}$ during the three change-detection tasks. Participants sat with head position fixed in a chinrest positioned $60 \mathrm{~cm}$ from the screen. Participants completed the complex span tasks on a separate computer in the same room. The only light in the room came from the light produced by the two computer monitors. Because we did not have any a priori predictions regarding eye-movements, we did not restrict eye movement other than instructing participants to bring their eyes to fixation during the fixation screens, and we did not analyze eye movements.

To obtain the primary dependent variable of pretrial pupil diameter, we first averaged pupil diameter across the right and left eyes. Then, we computed the average of this value across the 2-s fixation period for each trial. Missing data due to blinks and off-screen fixations were linearly interpolated unless all data points were missing for the trial. In that case, we excluded that trial from the analyses. If a participant was missing more than $50 \%$ of trials for a task, we excluded pupil data from that task from the analyses. Prior examinations have also examined phasic (task-evoked) pupillary responses (Robison \& Unsworth, 2019; Unsworth \& Robison, 2015, 2017b, 2018). But because the pre- and retrocueing trial types produced substantially different task-evoked pupil diameters with luminance confounds, and because participants made eye movements due to the spatial cues we restricted our analysis in the present study to pretrial (i.e., tonic) pupil diameter. see Supplemental Materials

We only included eye-tracking during the changedetection tasks for several reasons. First, these tasks have a relatively simple fixation-stimulus-delay-response sequence, allowing for a more straightforward analysis of pretrial pupil diameter and trial-to-trial changes therein. The complex span tasks have a rather complicated sequence of stimulus encoding periods, distractor task periods, and response screens, providing fewer "trials" within which we could examine moment-to-moment changes in arousal. However, we included both change-detection and complex span measures to generate a rather general estimate of WMC. We alternated between the change-detection and complex span tasks to give participants a break from being seated in the chinrest.

\section{Data analysis}

We used R (R Core Team, 2017) for all our analyses. Pupillometry data were aggregated offline to the level of analysis. We used several packages for data aggregation and analysis, including the tidyverse (Wickham, 2017), data.table (Dowle \& Srinivasan, 2018), zoo (Zeileis \& Grothendieck, 2005), lavaan (Rosseel, 2012), and apaTables (Stanley, 2018) packages. The manuscript was written in R Markdown using the papaja package (Aust \& Barth, 2018). All analysis scripts, data, and the script used to write the manuscript can be found on the Open Science Framework at the following url: https://osf.io/rhwqs/.

We screened the data for outliers by excluding any data points that fell outside $+/-3$ standard deviations of the mean. These data points were treated as missing in the descriptives, correlations, and factor analyses. One participant was removed listwise because the fire alarm sounded several times during their experimental session. A relatively larger number of participants are missing data for the Reading Span task because this task came last in the session and some participants ran out of time before reaching this task. This occurred for several reasons (e.g., arriving to the session late, taking longer than average to complete each task).

\section{Results}

Table 2 lists descriptive statistics, and Table 3 lists zeroorder correlations among all dependent measures. All measures were rather normally distributed (Kline, 2015) and had acceptable reliabilities. To estimate reliability for data from the visual arrays tasks, we used a SpearmanBrown split-half correlation. For the complex span tasks, we estimated a Cronbach's alpha from average number correct on each set size. 
Table 2 Descriptive statistics

\begin{tabular}{|c|c|c|c|c|c|c|}
\hline & $N$ & Mean & SD & Skew & Kurtosis & Reliability \\
\hline Operation span & 212 & 35.42 & 9.56 & -0.68 & 0.07 & 0.75 \\
\hline Symmetry span & 209 & 18.43 & 5.86 & -0.61 & 0.14 & 0.71 \\
\hline Reading span & 194 & 34.48 & 9.12 & -0.76 & 0.20 & 0.73 \\
\hline Accuracy - color & 213 & 0.77 & 0.12 & -0.85 & 0.20 & 0.91 \\
\hline Accuracy - orientation & 208 & 0.72 & 0.12 & -0.28 & -0.75 & 0.89 \\
\hline Accuracy - letter & 203 & 0.71 & 0.12 & -0.34 & -0.79 & 0.89 \\
\hline Mean pretrial pupil diameter - color & 193 & 4.68 & 0.68 & 0.26 & -0.48 & 1.00 \\
\hline Mean pretrial pupil diameter - orientation & 178 & 4.58 & 0.70 & 0.22 & -0.53 & 1.00 \\
\hline Mean pretrial pupil diameter - letter & 180 & 4.62 & 0.69 & 0.28 & -0.34 & 1.00 \\
\hline $\mathrm{CoV}$ pretrial pupil diameter - color & 192 & 0.07 & 0.02 & 0.68 & 0.29 & 0.92 \\
\hline $\mathrm{CoV}$ pretrial pupil diameter - orientation & 177 & 0.08 & 0.02 & 0.61 & -0.14 & 0.93 \\
\hline $\mathrm{CoV}$ pretrial pupil diameter - letter & 179 & 0.08 & 0.02 & 0.46 & -0.12 & 0.93 \\
\hline Lapse rate - color & 213 & 0.39 & 0.22 & 0.51 & -0.32 & NA \\
\hline Lapse rate - orientation & 208 & 0.46 & 0.29 & 0.31 & -1.13 & NA \\
\hline Lapse rate - letter & 203 & 0.42 & 0.31 & 0.42 & -1.07 & NA \\
\hline
\end{tabular}

Note. $\mathrm{SD}=$ standard deviation, $\mathrm{CoV}=$ coefficient of variation. Pupil diameter is in units of millimeters

In the following set of analyses, we specify several latent variable analyses to examine relationships among WMC, attentional lapse rate, and pupillary measures for average arousal and intraindividual variability in arousal. First, in a confirmatory factor analysis, we allowed the three complex span tasks to load onto a factor, the three visual arrays tasks to load onto a factor, mean pretrial pupil diameter from the three visual arrays tasks to load onto a factor, variability in pretrial pupil diameter from the visual arrays tasks to load onto a factor, and lapse rate from the visual arrays tasks to load onto a factor. The model fit the data well $\left(\chi^{2}(80)=159.49, \mathrm{CFI}=0.96, \mathrm{NNFI}=0.95, \mathrm{RMSEA}=0.07\right.$,

Table 3 Zero-order correlations

\begin{tabular}{|c|c|c|c|c|c|c|c|c|c|c|c|c|c|c|}
\hline & 1 & 2 & 3 & 4 & 5 & 6 & 7 & 8 & 9 & 10 & 11 & 12 & 13 & 14 \\
\hline 1. Operation span & - & & & & & & & & & & & & & \\
\hline 2. Symmetry span & $.45^{*}$ & - & & & & & & & & & & & & \\
\hline 3. Reading span & $.55^{*}$ & $.33^{*}$ & - & & & & & & & & & & & \\
\hline 4. Color accuracy & $.25^{*}$ & $.35^{*}$ & $.26^{*}$ & - & & & & & & & & & & \\
\hline 5. Orientation accuracy & $.34 *$ & $.39 *$ & $.28 *$ & $.63 *$ & - & & & & & & & & & \\
\hline 6. Letter accuracy & $.27 *$ & $.31 *$ & $.33 *$ & $.62 *$ & $.69^{*}$ & - & & & & & & & & \\
\hline 7. M pupil color & .08 & -.04 & .10 & .00 & .07 & .07 & - & & & & & & & \\
\hline 8. M pupil orientation & .05 & -.07 & .06 & -.01 & .05 & .03 & $.95^{*}$ & - & & & & & & \\
\hline 9. M pupil Letter & .05 & -.04 & .01 & .04 & .12 & .06 & $.92 *$ & $.97 *$ & - & & & & & \\
\hline 10. CoV pupil color & .01 & .09 & -.10 & -.07 & $-.16^{*}$ & -.13 & $-.24 *$ & $-.24 *$ & $-.19 *$ & - & & & & \\
\hline 11. CoV pupil orientation & -.10 & .05 & -.07 & -.06 & $-.29 *$ & $-.19 *$ & $-.20^{*}$ & $-.23 *$ & $-.23^{*}$ & $.69^{*}$ & - & & & \\
\hline 12. CoV pupil letter & -.08 & .02 & -.14 & -.12 & $-.29 *$ & $-.32 *$ & $-.20^{*}$ & $-.21 *$ & $-.24 *$ & $.65^{*}$ & $.74 *$ & - & & \\
\hline 13. Lapse color & -.06 & $-.17 *$ & -.09 & $-.33 *$ & $-.35^{*}$ & $-.30 *$ & $-.14 *$ & -.14 & -.10 & .12 & .07 & .10 & - & \\
\hline 14. Lapse orient & -.03 & $-.17 *$ & -.12 & $-.17 *$ & $-.32 *$ & $-.26^{*}$ & $-.15^{*}$ & $-.15^{*}$ & $-.21^{*}$ & .03 & $.22 *$ & .12 & $.62 *$ & - \\
\hline 15. Lapse letter & -.10 & $-.18^{*}$ & $-.16^{*}$ & $-.23 *$ & $-.31 *$ & $-.38^{*}$ & $-.17 *$ & $-.16^{*}$ & $-.17^{*}$ & .01 & .10 & $.18^{*}$ & $.60 *$ & $.76^{*}$ \\
\hline
\end{tabular}

Note M pupil = mean pretrial pupil diameter, $\mathrm{CoV}$ pupil $=$ coefficient of variation of pretrial pupil diameter, Lapse $=$ proportion of trials where participants reported an attentional lapse (external distraction, mind-wandering, or blank-mindedness). *indicates significant correlation at $\mathrm{p}<$ .05 . 


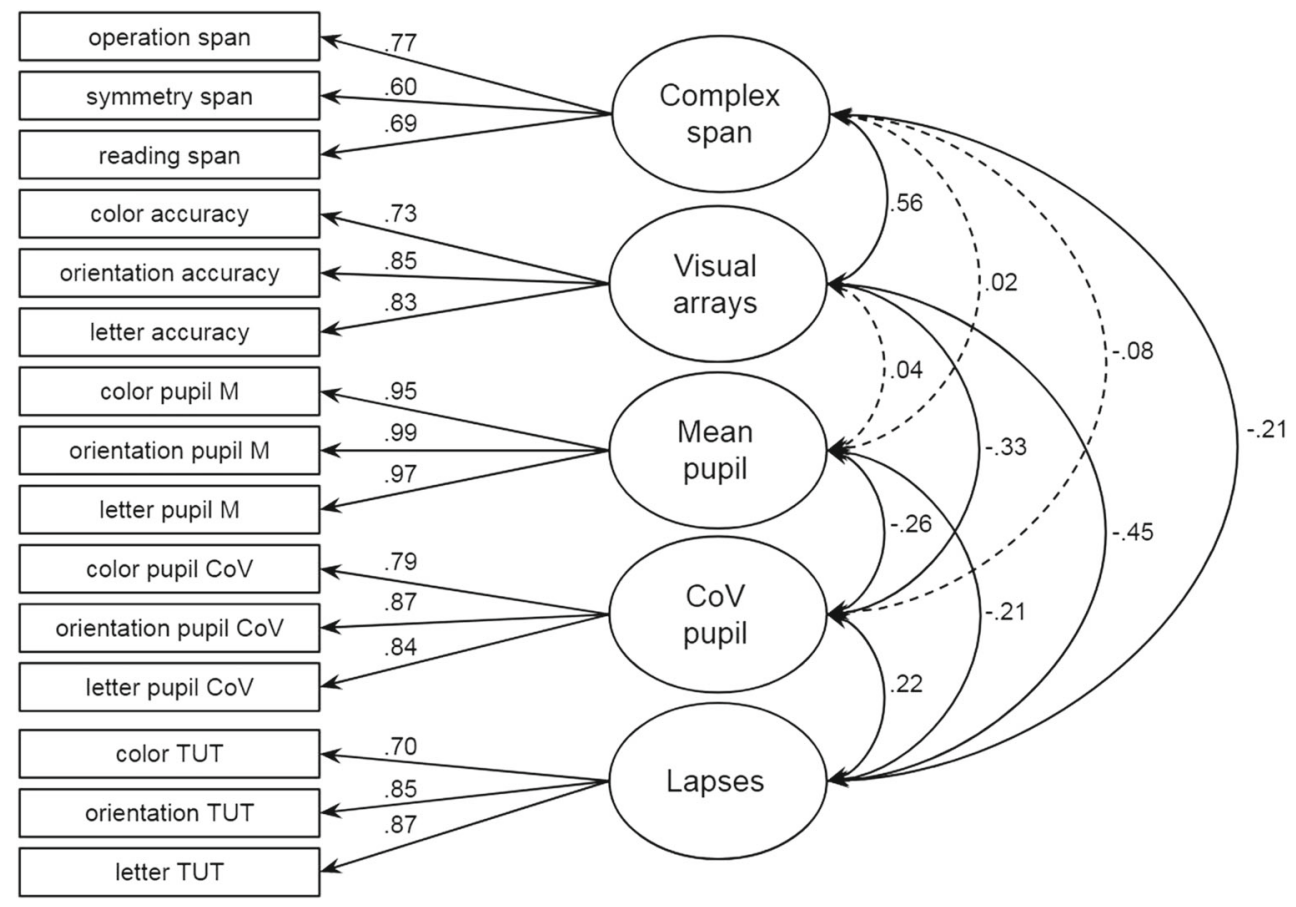

Fig. 5 Confirmatory factor analysis with working memory capacity, mean pretrial pupil, CoV of pretrial pupil, and lapse factors. Solid lines indicate significant paths at $p<.05$. Dotted lines indicate non-significant paths

SRMR $=0.04)^{1},{ }^{2}$ and is shown visually in Fig. 5. Several aspects of the model are notable. First, the complex span and visual arrays factors correlated, replicating prior research (Shipstead et al., 2014; Unsworth et al., 2014). However, these factors shared only about $31 \%$ of their variance. So while the complex span tasks and the visual arrays tasks tapped into some overlapping sources of variance, they also require some rather different abilities, as well. Second, the measures for mean pretrial pupil diameter and variability $(\mathrm{CoV})$ in pretrial pupil diameter loaded well onto their respective factors. This suggests that there is a task-general source of variance in these measures. Third, the complex span and visual arrays factors both negatively correlated with the Lapses factor. Thus people with higher WMC reported a lower rate of attentional lapses. Third, the mean pretrial pupil factor negatively correlated with the Lapses factor, suggesting people with higher overall arousal reported a lower rate of attentional lapses, consistent with a hypoarousal account. But this factor did not significantly correlate with either WM factor. Finally, the CoV pupil factor positively correlated with the Lapse factor and

\footnotetext{
${ }^{1} \mathrm{CFI}=$ comparative fit index, NNFI = non-normed fit index, RMSEA $=$ root mean squared error of approximation, SRMR = standardized root mean residual.

${ }^{2}$ The pattern of correlations among factors is largely the same when using $k$ as the manifest variable for the change-detection tasks rather than raw accuracy.
}

negatively correlated with the visual arrays factor. But it did not significantly correlate with the complex span factor. The fact that the visual arrays and $\mathrm{CoV}$ factors negatively correlated is consistent with the dysregulation account. However, the fact that the $\mathrm{CoV}$ and complex span factors did not correlate is inconsistent with this account, and does not replicate prior research (Unsworth \& Robison, 2017b). ${ }^{3}$

In the LC-NE account of individual differences in WMC and attention control, Unsworth and Robison argue that dysregulation of arousal manifests as attentional lapses that cause poor cognitive performance. To test this account, we specified two structural equation models in which dysregulation of arousal (pretrial pupil $\mathrm{CoV}$ ) and low arousal (mean pretrial pupil diameter) predict attentional lapses, which predicts visual array WM. Mean pupil and pupil $\mathrm{CoV}$ were allowed to have both direct and indirect effects on WMC. We specified separate models for complex span and visual arrays. The models are depicted in Figs. 6 and 7. CoV had a direct effect on visual arrays, but only

\footnotetext{
${ }^{3}$ Whereas Unsworth and Robison (2017b) used standard deviation as their measure of intraindividual variability in pretrial pupil diameter, we used coefficient of variation in the present study. Standard deviation and coefficient of variation are highly correlated measures (color: $r=0.86$, orientation: $r=0.87$, letter: $r=0.88$ ). If used in the latent variable analysis, the pattern of correlations remains the same, but the correlation between mean pretrial pupil diameter and standard deviation of pretrial pupil diameter switches sign from negative to positive.
} 


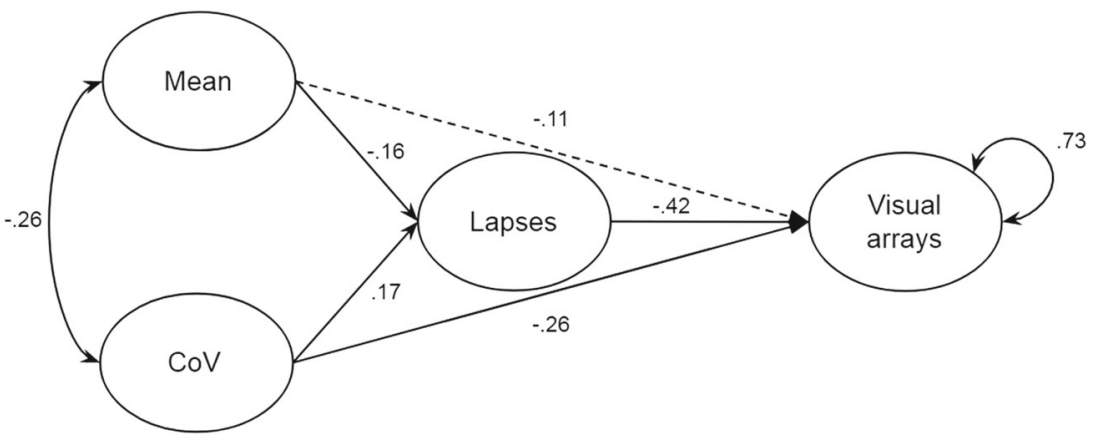

Fig. 6 Structural equation model testing the mediating effect of attentional lapses between arousal (mean pupil) arousal dysregulation (CoV pupil) and working memory capacity estimates from visual arrays tasks. Solid lines represent significant paths at $p<.05$. Dotted lines indicate non-significant paths

a marginally significant indirect effect through lapses $(b=$ $-.07, p=.05)$. Thus, attentional lapse rate only partially mediated the relation between arousal variability and visual arrays. Neither the direct effect of mean pupil diameter nor the indirect effect through lapses on visual arrays were significant. Collectively lapses, mean pretrial pupil diameter and variability in pretrial pupil diameter accounted for $27 \%$ of the variance in visual arrays performance. In the model predicting complex span (Fig. 4), none of the direct or indirect effects were significant, and collectively, the predictors only accounted for $3 \%$ of the variance in complex span performance.

Whereas Unsworth and Robison (2017b) used standard deviation as their measure of intraindividual variability in pretrial pupil diameter, we used coefficient of variation in the present study. Standard deviation and coefficient of variation are highly correlated measures (color: $r=0.86$, orientation: $r=0.87$, letter: $r=0.88$ ). If used in the latent variable analysis, the pattern of correlations remains the same, but the correlation between mean pretrial pupil diameter and standard deviation of pretrial pupil diameter switches sign from negative to positive.

\section{Blink rate and eye movements}

We additionally addressed two potential reasons for relations among variability in pretrial pupil diameter, visual arrays performance, and lapse rate. Specifically, it is possible that variability in pupil diameter is caused by individual differences in blink rate or eye movements. ${ }^{4}$ Thus, variability in pupil diameter may not be due to variation in arousal, but rather to the degree to which people move their eyes and blink during the tasks, both of which can affect pupillometry (Mathôt, Fabius, Van Heusden, \& Van der Stigchel, 2018). To examine this possibility, we computed

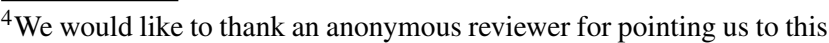
potential confound.
}

eye blink rate and fixation instability measure for each task. We categorized blinks as any period of data for which pupil diameter and gaze position for both eyes were missing for a time period of 100 to $500 \mathrm{~ms}$ (Jongkees \& Colzato, 2016; Peckham \& Johnson, 2016; Smilek, Carriere, \& Cheyne, 2010; Unsworth, Robison, \& Miller, 2019). We summed the number of blinks and divided by the task duration (in minutes) to compute a blink rate variable for each participant. For fixation instability we used intraindividual variability in gaze deviation from the center of the screen. Specifically, for each participant, we computed the instantaneous deviation from fixation using the $x$ - and $y$-coordinates of the right eye's gaze position at each sample. Then, we computed the standard deviation of these values across the 2000-ms fixation screen (higher standard deviation = greater fixation instability). We averaged this value across trials for each participant for each task. Then, we specified a confirmatory factor analysis similar to that in Fig. 5, but we added factors for blink rate and fixation instability. We allowed blinks from the color, orientation, and letter change-detection tasks to load onto a Blink Rate factor, and we allowed fixation instability estimates from those tasks to load on a Fixation Instability factor. We allowed these factors to correlate with the other factors in the model.

The model fit the data well $\left(\chi^{2}(168)=337.45\right.$, CFI $=0.94$, NNFI $=0.92$, RMSEA $=0.07$, SRMR $=0.05$ ). Neither the blink rate factor nor the fixation instability factor correlated significantly with the pupil variability factor (see Table 4). Thus, it did not appear that one of these factors was driving the relations among visual arrays performance, arousal variability, and lapse rate. But interestingly, the blink rate and fixation instability factors did significantly correlate with some other factors in the model. For example, blink rate positively correlated with visual arrays $(r=.28)$ and negatively correlated with lapse rate $(r=-.25)$. This suggests that people who blinked more often had higher estimates of visual working memory and reported fewer instances of task-unrelated thoughts. This is 


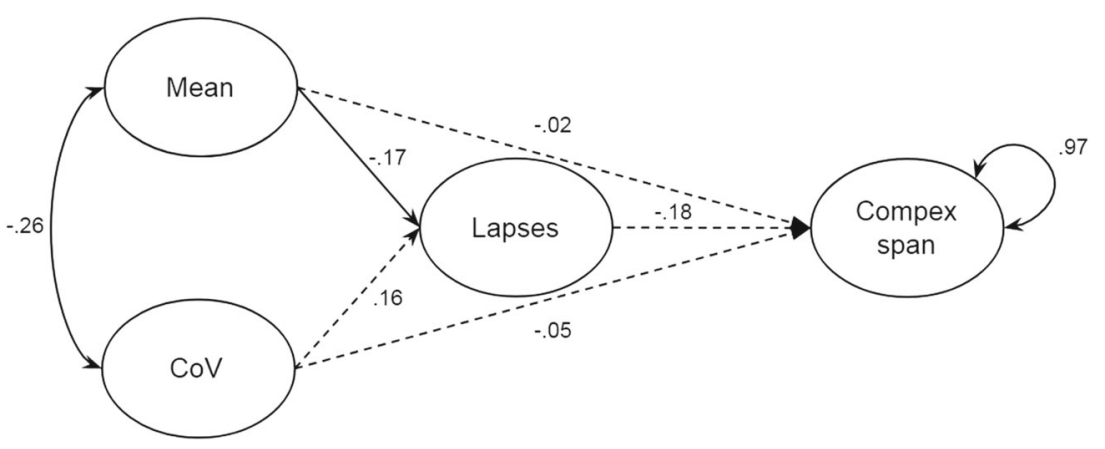

Fig. 7 Structural equation model testing the mediating effect of attentional lapses between arousal (mean pupil) arousal dysregulation (CoV pupil) and working memory capacity estimates from complex span tasks. Solid lines represent significant paths at $p<.05$. Dotted lines indicate non-significant paths

not a pattern that we predicted, but it begs replication and future investigation. Fixation instability also correlated with some of the other factors. It negatively correlated with both complex span $(r=-.21)$ and visual arrays $(r=-.50)$, and it positively correlated with lapse rate $(r=.25)$. Thus people whose eyes tended to stray from fixation during the fixation screen tended to show lower working memory capacity and more attentional lapses. Although we did not predict this pattern, this finding is consistent with prior research that has suggested that fixation stability might be an indicator of poor attention control (Munoz, Armstrong, Hampton, \& Moore, 2003; Unsworth et al., 2019). These findings were exploratory, and not the purpose of the present paper. But they offer interesting questions for future work.

\section{Discussion}

Recently, Unsworth and Robison (2017a) proposed a theory that one underlying source of variation that leads to individual differences in WMC is the relative functioning of the LC-NE system. They theorized that when this system breaks down, people have a tendency to fluctuate between states of arousal more readily, which can cause attentional lapses and poor cognitive performance. Unsworth and

Table 4 Interfactor correlations for model including blink rate and fixation instability

\begin{tabular}{lllllll}
\hline & 1 & 2 & 3 & 4 & 5 & 6 \\
\hline 1. Complex span & - & & & & & \\
2. Visual arrays & $.56^{*}$ & - & & & & \\
3. Pupil mean & .02 & .03 & - & & & \\
4. Pupil CoV & -.08 & $-.33^{*}$ & $-.26^{*}$ & - & & \\
5. Lapse rate & $-.21^{*}$ & $-.44^{*}$ & $-.20^{*}$ & $.21^{*}$ & - & \\
6. Blink rate & -.04 & $.28^{*}$ & -.07 & -.13 & $-.25^{*}$ & - \\
7. Fixation instability & $-.21^{*}$ & $-.50^{*}$ & .16 & .12 & $.25^{*}$ & -.11 \\
\hline
\end{tabular}

Note. $\mathrm{CoV}=$ coefficient of variation
Robison identified three potential mechanisms by which dysfunction of the LC-NE system could impact arousal and thus cognitive performance. The first is hypoarousal. It could be the case the low-WMC individuals are simply underaroused. If that were the case, people with low WMC should show smaller pupil diameters. Previously, Heitz et al. (2008) and Tsukahara et al. (2016) observed such a relationship. We did not observe that relationship in the present study. However, there was a significant negative correlation between attentional lapses and average pretrial pupil diameter, indicating that low-arousal participants did tend to experience more attentional lapses. Thus, there was one piece of evidence consistent with a hypoarousal hypothesis.

The second potential mechanism is hyperarousal. One piece of evidence consistent with this hypothesis was the fact that mean pretrial pupil diameter negatively correlated with WMC in Unsworth and Robison (2017b) latent variable analysis. Thus, low-WMC participants tended to have higher arousal overall in that study. However, we observed no evidence consistent with a hyperarousal hypothesis in the present study.

The third potential mechanism is dysregulation of arousal. If low-WMC people experience more shifts between states of low, intermediate, and high arousal more readily, then we should observe a negative correlation between intraindividual variability in pretrial pupil diameter and WMC and a positive correlation between intraindividual variability and attentional lapses. Here, we used the coefficient of variation $(\mathrm{CoV})$ as a measure of intraindividual variability. Indeed, the $\mathrm{CoV}$ factor negatively correlated with a factor representing performance on the visual arrays task and positively correlated with an attentional lapses factor. However, the $\mathrm{CoV}$ factor did not correlate with the complex span factor. Therefore, we observed several pieces of evidence consistent dysregulation hypothesis, but the fact that complex span did not correlate with the arousal regulation factor was inconsistent with this account. 
In our final analysis, we tested one specific prediction of the LC-NE account of working memory and attention-that the regulation of arousal affects cognition via attentional lapses (Unsworth \& Robison, 2017a). To do so, we specified two structural equation models in which attentional lapses were allowed to mediate the relations between the mean arousal and arousal dysregulation and WMC. Mean pretrial pupil diameter had neither a direct nor an indirect effect on either visual arrays or complex span factors. Thus, global arousal levels did little to explain variance in WMC. Dysregulation had a direct effect on the visual arrays factor, but not the complex span factor. Finally, the mediating effect of dysregulation on visual arrays via attentional lapses was not significant. Therefore, there was only partial support for that specific prediction.

At the outset, we had hoped to bring more data to light regarding one yet-unanswered research question. Why do people differ so substantially in WMC? What are the physiological and neural mechanisms by which the natural variation arises? To this end, we have made some strides. Prior work has examined the role of arousal regulation during measures of attention at the latent level (Unsworth \& Robison, 2017b), and other studies have examined arousal regulation during working memory tasks at the single-task level (Robison \& Unsworth, 2019; Unsworth \& Robison, 2015, 2018). This study served as an attempted replication and extension of the latent-variable approach taken by Unsworth and Robison (2017b) to examining relations among WMC, arousal regulation (measured via pupillometry), and attentional lapses. One weakness to this study is that we did not have any measures of higher-order cognition (e.g., reading comprehension, fluid intelligence) in order to test whether dysregulation of arousal also affects such abilities. In their study, Tsukahara et al. (2016) observed a relationship between fluid intelligence and baseline pupil size even after controlling for WMC and other potentially important variables (e.g., age, caffeine intake, sleep, etc.).

One major difference between the present study and Tsukahara et al. (2016) is that here and in Unsworth and Robison (2017b), pupil diameter was measured via pretrial measurements inserted into tasks. Tsukahara et al. (2016) measured pupil diameter during a task-free, preexperimental resting measurement. These are substantially different situations, and might account for why we did not observe an association between WMC and mean pupil diameter in the present study (but see Unsworth et al., 2019). In ongoing work, we are including both pre-experimental and pretrial measurements to see whether these two measures differentially correlate with cognitive abilities.

Several our of findings replicate prior studies that have demonstrated relations among arousal dysregulation and performance in visual arrays tasks (Robison \& Unsworth,
2019; Unsworth \& Robison, 2015), and that arousal dysregulation correlates with the tendency to experience attentional lapses (Unsworth \& Robison, 2017b). Therefore, arousal dysregulation rather reliably predicts both visual arrays working memory and the degree to which people experience lapses. However, we did not replicate the finding that measures of complex span WM correlate with measures of arousal dysregulation. Previously, Unsworth and Robison (2017b) observed this relationship. Here there were no significant associations between complex span WM and arousal regulation. There are several reasons why this could have been the case. First, Unsworth and Robison measured arousal during attention control (psychomotor vigilance and Stroop) tasks. Here, we measured arousal during visual WM tasks. Perhaps the differences across these two contexts lead to differential demands on arousal regulation and substantially change the nature of the relation between WMC and arousal regulation. The visual arrays tasks have a repetitive stimulus-response (or stimulus-delayresponse) structure that repeats over many trials. Whereas complex span tasks have a more complicated and less repetitive structure. It is possible that arousal regulation is more important for performance in situations that require consistent attention to a repetitive task. Another possibility is that visual arrays tasks, especially ones that add additional selectivity requirements like those in the present study, lean more heavily on an individual's attentional system than their memory system (Martin, Tsukahara, Draheim, Mashburn, \& Engle, 2019; Robison et al., 2018; Robison \& Unsworth 2017; Shipstead et al., 2012, 2014, 2015). If that is the case, then the present results largely replicate findings by Unsworth and Robison (2017b) that variability in arousal is an underlying difference that correlates with one's ability to control their attention. This hypothesis will require future investigation. Second, the fact that we observed a relationship between arousal dysregulation and visual arrays performance, but not complex span, could be due to the fact that arousal regulation was measured during the visual arrays tasks. Therefore, there is shared method and temporal variance between these measures. However, the complex span and visual arrays tasks were completed during the same session and in alternating fashion. Therefore, the extent to which temporal factors (e.g., alertness, motivation) affect arousal regulation, they should have affected visual arrays and complex span tasks about equally. We chose to measure arousal regulation during the visual arrays tasks because they provide more "trials" by which we can measure both mean arousal and variability in arousal. Complex span tasks are just that-complex-and they make the measurement of pupil diameter (i.e., arousal) considerably less straightforward. Thus it is still not entirely clear why arousal regulation should correlate with visual arrays task performance, but less so with complex span task 
performance. We are currently following up on this finding in ongoing research.

Earlier, we noted that one influential theory regarding the cause of individual differences in WMC is differential functioning of the DLPFC (Kane \& Engle, 2002). We should note that it is possible that this is indeed a source of variation. While we posit a different mechanismarousal dysregulation via differential LC-NE functioningthese mechanisms are not mutually exclusive. We cannot rule out that individuals who experience more variability in arousal do not also have relatively weaker DLPFC functioning. It could also be the case that some people have relatively low WMC because of poor arousal regulation, whereas others have low WMC because of poor DLPFC functioning. With the present data, we cannot specifically address the DLPFC theory. But because we know there are physiological links between pupil diameter and LC$\mathrm{NE}$ functioning, we can be relatively certain that the inter- and intraindividual variability in pupillary measures indexes, to a reasonable extent, differential activity of the LC-NE system. It is also the case that NE is not the only neuromodulator that affects cognition. Indeed, other systems likely work in concert with the LC-NE system (e.g., the dopaminergic and cholinergic systems) to give rise to the complex set of computations required in any situation that places demands on working memory and attention (Cools \& D'Esposito 2011, Sara, 2009; Sarter, Givens, \& Bruno, 2001; Sawaguchi \& Goldman-Rakic, 1991.

Although it was not an aim of the present study at the outset, an anonymous reviewer suggested we examine whether other individual differences (eye blink rate, fixation instability) might be driving the correlations we observed with pupil diameter. Both blinking and eye movements can affect pupillometry, so this is a valid concern (Mathôt et al., 2018). Therefore, we computed an eye blink rate measure for each task, as well as a measure of fixation instability. Interestingly, we were able to specify factors for these measures at the latent level, indicating there is some degree of trait stability to the measures. But neither the blink rate factor nor the fixation instability factor significantly correlated with the pupil variability factor. Therefore, we do not believe these other factors were driving our findings. However, we did observe some significant correlations among eye blink rate, fixation instability, and the other factors. Specifically, eye blink rate positively correlated with visual arrays performance, whereas fixation instability negatively correlated with visual arrays. Eye blink rate and fixation instability also correlated with lapse rate, but in opposite directions. People who reported more attentional lapses tended to blink less often but have more fixation instability. Eye blink rate and fixation instability did not significantly correlate.
It is important to note that we did not predict these patterns, and these analyses were completed in an exploratory manner. But they are consistent with some prior work. For example, prior studies have shown that fixation instability is higher among individuals with poor attention control (Unsworth et al., 2019), individuals with attention deficit-hyperactivity disorder (Munoz et al., 2003), autism spectrum disorders (Shirama, Kanai, Kato, \& Kashino, 2016), schizophrenia, and obsessive-compulsive disorders (Barton, Pandita, Thakkar, Goff, \& Manoach, 2008; Benson et al., 2012; Damilou, Apostolakis, Thrapsanioti, Theleritis, \& Smyrnis, 2016), individuals high in trait anxiety (Laretzaki et al., 2011), and individuals low in intelligence (Smyrnis et al., 2004). Thus, if fixation instability is a rather general indicator of poor cognitive control, that would be consistent with our observation that it negatively correlates with working memory capacity and positively with attentional lapses. It has also been suggested that eye blink rate can be used as an indirect measure of activity in the dopaminergic system (Colzato, Wildenberg, Wouwe, Pannebakker, \& Hommel, 2009; Jongkees \& Colzato, 2016; Peckham \& Johnson, 2016), and dopamine has been implicated in the regulation of working memory (e.g., Sawaguchi \& Goldman-Rakic, 1991; Braver \& Cohen, 2000; Cools \& D'Esposito, 2011). Previously, Unsworth et al. (2019) did not find any relations between spontaneous eye blink rate and working memory capacity or attention control abilities. However, their study used spontaneous eye blink rate during a pre-experimental resting period, whereas these measures came within the context of a working memory task. If the relation between blink rate and task performance can be replicated, and there is reason to believe eye blink rate is an indirect yet valid measure of dopaminergic system activity, this opens doors for future research combining pupillometry and blink rate to study the shared and unique influences of the dopaminergic and noradrenergic systems on individual differences in working memory capacity.

\section{Conclusions}

We sought to test whether three different mechanisms would account for individual differences in working memory capacity and the ability to resist attentional lapses. However, we found only mixed evidence for the two of the mechanisms (hypoarousal and arousal dysregulation). Three major pieces of evidence were consistent with an arousal dysregulation account: people who demonstrated lower visual arrays performance showed more arousal dysregulation, 2) people who self-reported more attentional lapses showed more arousal dysregulation, and 3) people who self-reported more attentional lapses demonstrated lower visual arrays performance and lower complex span 
performance. These are all predictions made by Unsworth and Robison (2017a) in their LC-NE theory of working memory and attention control. However, one piece of evidence was lacking in the current study. Complex span performance did not correlate with arousal regulation, as it did in one prior study Unsworth and Robison (2017b). Despite a moderate latent correlation between complex span and visual arrays in a factor analysis $(r=.56)$, they showed different correlations with an arousal regulation factor. Thus, future research will necessary to further delineate the nature of individual differences in various cognitive abilities and how they are impacted by arousal, and under what situations arousal regulation is most important for optimal cognitive performance.

\section{Open practices statement}

We have made all of our data for the present study available to the public on the Open Science Framework (https://osf.io/ rhwqs/). An R markdown document containing all analyses is on the Open Science Framework under the same URL.

\section{References}

Andrews-Hanna, J. R., Smallwood, J., \& Spreng, R. N. (2014). The default network and self-generated thought: Component processes, dynamic control, and clinical relevance. Annals of the New York Academy of Sciences, 1316, 29-52.

Aston-Jones, G., \& Cohen, J. D. (2005). An integrative theory of locus coeruleus-norepinephrine function: Adaptive gain and optimal performance. Annual Reviews of Neuroscience, 28, 403-450. https://doi.org/10.1146/annurev.neuro.28.061604.135709

Aust, F., \& Barth, M. (2018). papaja: Create APA manuscripts with R Markdown. Retrieved from https://github.com/crsh/papaja.

Barton, J. J., Pandita, M., Thakkar, K., Goff, D. C., \& Manoach, D. S. (2008). The relation between antisaccade errors, fixation stability and prosaccade errors in schizophrenia. Experimental Brain Research, 186, 273-282.

Benson, P. J., Beedie, S. A., Shephard, E., Giegling, I., Rujescu, D., \& Clair, D. S. (2012). Simple viewing tests can detect eye movement abnormalities that distinguish schizophrenia cases from controls with exceptional accuracy. Biological Psychiatry, 72, 716-724.

Berridge, C. W., \& Waterhouse, B. D. (2003). The locus coeruleusnoradrenergic system: Modulation of behavioral state and statedependent cognitive processes. Brain Research Reviews, 42, 33 84

Braver, T. S., \& Cohen, J. D. (2000). On the control of control: the role of dopamine in regulating prefrontal function and working memory. Control of Cognitive Processes: Attention and Performance XVIII, 713-737.

Cohen, J. D., Aston-Jones, G., \& Gilzenrat, M. S. (2004). A systemslevel perspective on attention and cognitive control: Guided activation, adaptive gating, conflict monitoring, and exploitation versus exploration. In Posner, M. I. (Ed.) Cognitive neuroscience of attention, (pp. 71-90): Guilford Press.

Colzato, L. S., Wildenberg, W. P., van Wouwe, N. C., Pannebakker, M. M., \& Hommel, B. (2009). Van den Dopamine and inhibitory action control: Evidence from spontaneous eye blink rates. Experimental Brain Research, 196, 467-474.

Conway, A. R., Cowan, N., Bunting, M. F., Therriault, D. J., \& Minkoff, S. R. (2002). A latent variable analysis of working memory capacity, short-term memory capacity, processing speed, and general fluid intelligence. Intelligence, 30, 163-183.

Cools, R., \& D'Esposito, M. (2011). Inverted-u-shaped dopamine actions on human working memory and cognitive control. Biological Psychiatry, 69, e113-e125.

Corbetta, M., \& Shulman, G. L. (2002). Control of goal-directed and stimulus-driven attention in the brain. Nature Reviews Neuroscience, 3, 201.

Cowan, N. (2001). Metatheory of storage capacity limits. Behavioral and Brain Sciences, 24, 154-176.

Cowan, N., Elliott, E. M., Saults, J. S., Morey, C. C., Mattox, S., Hismjatullina, A., \& Conway, A. R. (2005). On the capacity of attention: Its estimation and its role in working memory and cognitive aptitudes. Cognitive Psychology, 51, 42-100.

Damilou, A., Apostolakis, S., Thrapsanioti, E., Theleritis, C., \& Smyrnis, N. (2016). Shared and distinct oculomotor function deficits in schizophrenia and obsessive compulsive disorder. Psychophysiology, 53, 796-805.

Daneman, M., \& Carpenter, P. A. (1980). Individual differences in working memory and reading. Journal of Verbal Learning and Verbal Behavior, 19, 450-466.

Dosenbach, N. U., Fair, D. A., Cohen, A. L., Schlaggar, B. L., \& Petersen, S. E. (2008). A dual-networks architecture of top-down control. Trends in Cognitive Sciences, 12, 99-105.

Dowle, M., \& Srinivasan, A. (2018). Data.table: Extension of 'data.frame'. Retrieved from https://CRAN.R-project.org/ package $=$ data.table.

Eldar, E., Cohen, J. D., \& Niv, Y. (2013). The effects of neural gain on attention and learning. Nature Neuroscience, 16, 1146.

Engle, R. W., \& Kane, M. J. (2004). Executive attention, working memory capacity, and a two-factor theory of cognitive control. Psychology of Learning and Motivation, 44, 145-200.

Engle, R. W., Tuholski, S. W., Laughlin, J. E., \& Conway, A. R. (1999). Working memory, short-term memory, and general fluid intelligence:, A latent-variable approach. Journal of Experimental Psychology: General, 128, 309.

Fox, M. D., Snyder, A. Z., Vincent, J. L., Corbetta, M., Van Essen, D. C., \& Raichle, M. E. (2005). The human brain is intrinsically organized into dynamic, anticorrelated functional networks. Proceedings of the National Academy of Sciences, 102, 9673-9678.

Fukuda, K., Vogel, E., Mayr, U., \& Awh, E. (2010). Quantity, not quality: the relationship between fluid intelligence and working memory capacity. Psychonomic Bulletin \& Review, 17, 673-679.

Gilzenrat, M. S., Nieuwenhuis, S., Jepma, M., \& Cohen, J. D. (2010). Pupil diameter tracks changes in control state predicted by the adaptive gain theory of locus coeruleus function. Cognitive, Affective, \& Behavioral Neuroscience, 10, 252-269. https://doi.org/10.3758/CABN.10.2.252

Heitz, R. P., Schrock, J. C., Payne, T. W., \& Engle, R. W. (2008). Effects of incentive on working memory capacity: Behavioral and pupillometric data. Psychophysiology, 45, 119129. https://doi.org/10.1111/j.1469-8986.2007.00605.x

Jongkees, B. J., \& Colzato, L. S. (2016). Spontaneous eye blink rate as predictor of dopamine-related cognitive function-a review. Neuroscience \& Biobehavioral Reviews, 71, 58-82.

Joshi, S., Li, Y., Kalwani, R. M., \& Gold, J. I. (2016). Relationships between pupil diameter and neuronal activity in the locus coeruleus, colliculi, and cingulate cortex. Neuron, 89, 221-234.

Kane, M. J., Bleckley, M. K., Conway, A. R., \& Engle, R. W. (2001). A controlled-attention view of working-memory capacity. Journal of Experimental Psychology:, General, 130, 169-183. 
Kane, M. J., Brown, L. H., McVay, J. C., Silvia, P. J., Myin-Germeys, I., \& Kwapil, T. R. (2007). For whom the mind wanders, and when: an experience-sampling study of working memory and executive control in daily life. Psychological Science, 18, 614-621.

Kane, M. J., \& Engle, R. W. (2002). The role of prefrontal cortex in working-memory capacity, executive attention, and general fluid intelligence: an individual-differences perspective. Psychonomic Bulletin \& Review, 9, 637-671.

Kane, M. J., Hambrick, D. Z., Tuholski, S. W., Wilhelm, O., Payne, T. W., \& Engle, R. W. (2004). The generality of working memory capacity:, A latent-variable approach to verbal and visuospatial memory span and reasoning. Journal of Experimental Psychology: General, 133, 189-217.

Kane, M. J., Meier, M. E., Smeekens, B. A., Gross, G. M., Chun, C. A., Silvia, P. J., \& Kwapil, T. R. (2016). Individual differences in the executive control of attention, memory, and thought, and their associations with schizotypy. Journal of Experimental Psychology:, General, 145, 1017-1048.

Kessler, R. C., Adler, L., Ames, M., Demler, O., Faraone, S., Hiripi, E., \& et al. (2005). The world health organization adult ADHD self-report scale (ASRS):, A short screening scale for use in the general population. Psychological Medicine, 35, 245-256.

Kline, R. B. (2015). Principles and practice of structural equation modeling. Guilford publications.

Konishi, M., McLaren, D. G., Engen, H., \& Smallwood, J. (2015). Shaped by the past: the default mode network supports cognition that is independent of immediate perceptual input. PloS One, 10, e0132209.

Kyllonen, P. C., \& Christal, R. E. (1990). Reasoning ability is (little more than) working-memory capacity?!. Intelligence, 14, 389433.

Kyllonen, P. C., \& Stephens, D. L. (1990). Cognitive abilities as determinants of success in acquiring logic skill. Learning and Individual Differences, 2, 129-160.

Laretzaki, G., Plainis, S., Vrettos, I., Chrisoulakis, A., Pallikaris, I., \& Bitsios, P. (2011). Threat and trait anxiety affect stability of gaze fixation. Biological Psychology, 86, 330-336.

Luck, S. J., \& Vogel, E. K. (1997). The capacity of visual working memory for features and conjunctions. Nature, 390, 279-281.

Martin, J., Tsukahara, J. S., Draheim, C., Mashburn, C., \& Engle, R. W. (2019). The visual arrays task... visual storage capacity or attention control?.

Mathôt, S., Fabius, J., Van Heusden, E., \& Van der Stigchel, S. (2018). Safe and sensible preprocessing and baseline correction of pupil-size data. Behavior Research Methods, 50(1), 94-106.

McVay, J. C., \& Kane, M. J. (2012a). Drifting from slow to "d'oh!": Working memory capacity and mind wandering predict extreme reaction times and executive control errors. Journal of Experimental Psychology: Learning, Memory, and Cognition, 38, 525-549.

McVay, J. C., \& Kane, M. J. (2012b). Why does working memory capacity predict variation in reading comprehension? on the influence of mind wandering and executive attention. Journal of Experimental Psychology:, General, 141, 302. https://doi.org/10.1037/a0025250

Menon, V., \& Uddin, L. Q. (2010). Saliency, switching, attention and control: a network model of insula function. Brain Structure and Function, 214, 655-667.

Miller, E. K., \& Cohen, J. D. (2001). An integrative theory of prefrontal cortex function. Annual Review of Neuroscience, 24, 167-202.

Munoz, D. P., Armstrong, I. T., Hampton, K. A., \& Moore, K. D. (2003). Altered control of visual fixation and saccadic eye movements in attention-deficit hyperactivity disorder. Journal of Neurophysiology, 90(1), 503-514.
Peckham, A. D., \& Johnson, S. L. (2016). Spontaneous eye-blink rate as an index of reward responsivity: Validation and links to bipolar disorder. Clinical Psychological Science, 4, 451-463.

Petersen, S. E., \& Posner, M. I. (2012). The attention system of the human brain: 20 years after. Annual Review of Neuroscience, 35, 73-89.

Raichle, M. E., MacLeod, A. M., Snyder, A. Z., Powers, W. J., Gusnard, D. A., \& Shulman, G. L. (2001). A default mode of brain function. Proceedings of the National Academy of Sciences, 98, 676-682.

R Core Team (2017). R: A language and environment for statistical computing. Vienna, Austria: R Foundation for Statistical Computing. Retrieved from https://www.R-project.org/.

Robison, M. K., Brewer, G. A., \& Unsworth, N. (2020). A multicomponent analysis of working memory capacity and attention control. Manuscript in Preparation.

Robison, M. K., Gath, K. I., \& Unsworth, N. (2017). The neurotic wandering mind: an individual differences investigation of neuroticism, mind-wandering, and executive control. The Quarterly Journal of Experimental Psychology, 70, 649663.

Robison, M. K., Miller, A. L., \& Unsworth, N. (2018). Individual differences in working memory capacity and filtering. Journal of Experimental Psychology:, Human Perception and Performance, 44, 1038-1053.

Robison, M. K., \& Unsworth, N. (2015). Working memory capacity offers resistance to mind-wandering and external distraction in a context-specific manner. Applied Cognitive Psychology, 29, 680-690.

Robison, M. K., \& Unsworth, N. (2017). Variation in the use of cues to guide visual working memory. Attention, Perception, \& Psychophysics, 79, 1652-1665.

Robison, M. K., \& Unsworth, N. (2018). Cognitive and contextual correlates of spontaneous and deliberate mind-wandering. Journal of Experimental Psychology: Learning, Memory, and Cognition, 44, 85. https://doi.org/10.1037/xlm0000444

Robison, M. K., \& Unsworth, N. (2019). Pupillometry tracks fluctuations in working memory performance. Attention, Perception, \& Psychophysics, 81, 407-419. https://doi.org/10.3758/s13414-0181618-4

Rosseel, Y. (2012). lavaan: An R package for structural equation modeling. Journal of Statistical Software, 48, 1-36. Retrieved from http://www.jstatsoft.org/v48/i02/.

Samuels, E. R., \& Szabadi, E. (2008). Functional neuroanatomy of the noradrenergic locus coeruleus: Its roles in the regulation of arousal and autonomic function part I: Principles of functional organisation. Current Neuropharmacology, 6, 235-253.

Sara, S. J. (2009). The locus coeruleus and noradrenergic modulation of cognition. Nature Reviews Neuroscience, 10, 211-223. https://doi.org/10.1038/nrn2573

Sarter, M., Givens, B., \& Bruno, J. P. (2001). The cognitive neuroscience of sustained attention: Where top-down meets bottom-up. Brain Research Reviews, 35, 146-160.

Sawaguchi, T., \& Goldman-Rakic, P. S. (1991). D1 dopamine receptors in prefrontal cortex: Involvement in working memory. Science, 251, 947-950.

Schmiedek, F., Oberauer, K., Wilhelm, O., Süß, H.-M., \& Wittmann, W. W. (2007). Individual differences in components of reaction time distributions and their relations to working memory and intelligence. Journal of Experimental Psychology:, General, 136, 414-429.

Seeley, W. W., Menon, V., Schatzberg, A. F., Keller, J., Glover, G. H., Kenna, H., \& Greicius, M. D. (2007). Dissociable intrinsic connectivity networks for salience processing and executive control. Journal of Neuroscience, 27, 2349-2356. 
Shipstead, Z., Harrison, T. L., \& Engle, R. W. (2015). Working memory capacity and the scope and control of attention. Attention, Perception, \& Psychophysics, 77, 1863-1880.

Shipstead, Z., Lindsey, D. R., Marshall, R. L., \& Engle, R. W. (2014). The mechanisms of working memory capacity: Primary memory, secondary memory, and attention control. Journal of Memory and Language, 72, 116-141.

Shipstead, Z., Redick, T. S., Hicks, K. L., \& Engle, R. W. (2012). The scope and control of attention as separate aspects of working memory. Memory, 20, 608-628.

Shirama, A., Kanai, C., Kato, N., \& Kashino, M. (2016). Ocular fixation abnormality in patients with autism spectrum disorder. Journal of Autism and Developmental Disorders, 46, 16131622.

Smallwood, J., Tipper, C., Brown, K., Baird, B., Engen, H., Michaels, J. R., \& Schooler, J. W. (2013). Escaping the here and now: Evidence for a role of the default mode network in perceptually decoupled thought. NeuroImage, 69, 120-125.

Smilek, D., Carriere, J. S., \& Cheyne, J. A. (2010). Out of mind, out of sight: Eye blinking as indicator and embodiment of mind wandering. Psychological Science, 21, 786-789.

Smyrnis, N., Kattoulas, E., Evdokimidis, I., Stefanis, N., Avramopoulos, D., Pantes, G., \& Stefanis, C. (2004). Active eye fixation performance in 940 young men:, Effects of IQ, schizotypy, anxiety and depression. Experimental Brain Research, 156, 1-10.

Spreng, R. N., DuPre, E., Selarka, D., Garcia, J., Gojkovic, S., Mildner, J., \& Turner, G. R. (2014). Goal-congruent default network activity facilitates cognitive control. Journal of Neuroscience, 34 , $14108-14114$.

Stanley, D. (2018). ApaTables: Create American Psychological Association (APA) style tables. Retrieved from https://CRAN. R-project.org/package $=$ apaTables.

Szabadi, E. (2013). Functional neuroanatomy of the central noradrenergic system. Journal of Psychopharmacology, 27, 659-693.

Tsukahara, J. S., Harrison, T. L., \& Engle, R. W. (2016). The relationship between baseline pupil size and intelligence. Cognitive Psychology, 91, 109-123.

Unsworth, N. (2016). The many facets of individual differences in working memory capacity. In Ross, B. (Ed.) Psychology of learning and motivation, (Vol. 65, pp. 1-46): Elsevier.

Unsworth, N., Brewer, G. A., \& Spillers, G. J. (2012a). Variation in cognitive failures: an individual differences investigation of everyday attention and memory failures. Journal of Memory and Language, 67, 1-16.

Unsworth, N., \& Engle, R. W. (2005). Individual differences in working memory capacity and learning: Evidence from the serial reaction time task. Memory \& Cognition, 33, 213-220.

Unsworth, N., \& Engle, R. W. (2007a). On the division of short-term and working memory: an examination of simple and complex span and their relation to higher order abilities. Psychological Bulletin, 133, 1038-1066.

Unsworth, N., \& Engle, R. W. (2007b). The nature of individual differences in working memory capacity: Active maintenance in primary memory and controlled search from secondary memory. Psychological Review, 114, 104-132.

Unsworth, N., Fukuda, K., Awh, E., \& Vogel, E. K. (2014). Working memory and fluid intelligence: Capacity, attention control, and secondary memory retrieval. Cognitive Psychology, 71, 1-26. Retrieved https://doi.org/10.1016/j.cogpsych.2014.01.003.

Unsworth, N., Heitz, R. P., Schrock, J. C., \& Engle, R. W. (2005). An automated version of the operation span task. Behavior Research Methods, 37, 498-505.

Unsworth, N., \& McMillan, B. D. (2013). Mind wandering and reading comprehension: Examining the roles of working memory capacity, interest, motivation, and topic experience. Journal of Experimental Psychology: Learning, Memory, and Cognition, 39, 832-842.

Unsworth, N., \& McMillan, B. D. (2014). Similarities and differences between mind-wandering and external distraction: a latent variable analysis of lapses of attention and their relation to cognitive abilities. Acta Psychologica, 150, 14-25.

Unsworth, N., McMillan, B. D., Brewer, G. A., \& Spillers, G. J. (2012b). Everyday attention failures: An individual differences investigation. Journal of Experimental Psychology: Learning, Memory, and Cognition, 38, 1765.

Unsworth, N., Redick, T. S., Heitz, R. P., Broadway, J. M., \& Engle, R. W. (2009). Complex working memory span tasks and higher-order cognition: a latent-variable analysis of the relationship between processing and storage. Memory, 17, 635654. https://doi.org/10.1080/09658210902998047

Unsworth, N., Redick, T. S., Lakey, C. E., \& Young, D. L. (2010). Lapses in sustained attention and their relation to executive control and fluid abilities: an individual differences investigation. Intelligence, 38, 111-122. https://doi.org/10.1016/j.intell.2009.08.002

Unsworth, N., \& Robison, M. K. (2015). Individual differences in the allocation of attention to items in working memory: Evidence from pupillometry. Psychonomic Bulletin \& Review, 22, 757-765. https://doi.org/10.3758/s13423-014-0747-6

Unsworth, N., \& Robison, M. K. (2017a). A locus coeruleusnorepinephrine account of individual differences in working memory capacity and attention control. Psychonomic Bulletin \& Review, 24, 1282-1311. https://doi.org/10.3758/s13423-016$1220-5$

Unsworth, N., \& Robison, M. K. (2017b). The importance of arousal for variation in working memory capacity and attention control: A latent variable pupillometry study. Journal of Experimental Psychology: Learning, Memory, and Cognition, 43, 1962-1987. https://doi.org/10.1037/xlm0000421

Unsworth, N., \& Robison, M. K. (2018). Tracking working memory maintenance with pupillometry. Attention, Perception, \& Psychophysics, 80, 461-484. https://doi.org/10.3758/s13414-0171455-x

Unsworth, N., Robison, M. K., \& Miller, A. L. (2019). Individual differences in baseline oculometrics: Examining variation in baseline pupil diameter, spontaneous eye blink rate, and fixation stability. Cognitive, Affective, \& Behavioral Neuroscience, 19, 1074-1093.

Varazzani, C., San-Galli, A., Gilardeau, S., \& Bouret, S. (2015). Noradrenaline and dopamine neurons in the reward/effort trade-off: a direct electrophysiological comparison in behaving monkeys. Journal of Neuroscience, 35, 7866-7877.

Vatansever, D., Menon, D. K., Manktelow, A. E., Sahakian, B. J., \& Stamatakis, E. A. (2015). Default mode dynamics for global functional integration. Journal of Neuroscience, 35, 15254-15262.

Vincent, J. L., Kahn, I., Snyder, A. Z., Raichle, M. E., \& Buckner, R. L. (2008). Evidence for a frontoparietal control system revealed by intrinsic functional connectivity. Journal of Neurophysiology, 100, 3328-3342.

Vogel, E. K., McCollough, A. W., \& Machizawa, M. G. (2005). Neural measures reveal individual differences in controlling access to working memory. Nature, 438, 500-503.

Wickham, H. (2017). Tidyverse: Easily install and load the 'tidyverse'. Retrieved from https://CRAN.R-project.org/package=tidyverse.

Zeileis, A., \& Grothendieck, G. (2005). Zoo: s3 infrastructure for regular and irregular time series. Journal of Statistical Software, 14,1-27. https://doi.org/10.18637/jss.v014.i06

Publisher's note Springer Nature remains neutral with regard to jurisdictional claims in published maps and institutional affiliations. 